\title{
Tinea faciei in a central Portuguese hospital: A 9-year survey
}

\author{
Ana Borges (iD | Ana Brasileiro (i) | Célia Galhardas | Margarida Apetato
}

Department of Dermatology and Venereology, Hospital Santo António dos Capuchos, Centro Hospitalar de Lisboa

Central, Lisbon, Portugal

\section{Correspondence}

Ana Borges, Department of Dermatology and Venereology, Hospital Santo António dos Capuchos, Centro Hospitalar de Lisboa Central, Lisbon, Portugal.

Email: asofiarborges@gmail.com

\begin{abstract}
Summary
Tinea faciei is a relatively uncommon dermatophytosis that affects the glabrous skin of the face. The aim of this study was to analyse the epidemiologic, clinical and mycological features of tinea faciei cases diagnosed at the Dermatology and Venereology Department of Hospital Santo António dos Capuchos (Lisbon, Portugal). Consecutive cases diagnosed between 2008 and 2016 were studied retrospectively. A total of 72 tinea faciei cases have been diagnosed, involving 37 male and 35 female, aged between 8 months and 86 years. The majority were observed in patients younger than 12 years of age (59.72\%). Anthropophilic isolates (mainly Microsporum audouinii, Trichophyton soudanense and Trichophyton rubrum) accounted for $75.7 \%$ of the identified dermatophytes. One quarter of the patients were also affected by dermatophytosis in other areas, such as the scalp. Only 10 cases were previously treated with topical steroids due to misdiagnosis. Most patients were treated with topical and systemic antifungal therapy with total resolution of skin lesions, without relapse or side effects. In contrast to other European studies, anthropophilic dermatophytes were the main causative agents of tinea faciei. As previously described to tinea capitis, this result is probably due to changes in the epidemiology of dermatophytes worldwide.
\end{abstract}

\section{KEYWORDS}

anthropophilic dermatophytes, tinea faciei, tinea incognito

\section{1 | INTRODUCTION}

Tinea faciei is a superficial dermatophyte infection of the facial nonbearded regions. ${ }^{1}$ Concerning the epidemiology, 2 peaks of incidence are to be considered: one in children, in whom it occurs mainly as a result of contact with pets, and the other in adults, resulting from occupational exposure or leisure activities, ${ }^{1-3}$ mostly contaminated fomites. Tinea faciei is often confused with other dermatoses, as fungal infections are more frequent in other anatomic locations. ${ }^{1}$ Additionally, the differential diagnosis with other dermatoses such as lupus erythematosus, ${ }^{4}$ rosacea ${ }^{5}$ and granuloma annulare ${ }^{6}$ is not always straightforward, as atypical features are more common in this location, possibly related with the complex anatomy of the face. ${ }^{1}$ As a result of misdiagnosis, these lesions are often treated with topical steroids or calcineurin inhibitors, which can also result in changes in the clinical appearance. ${ }^{7}$

Concerning the aetiology, the causative agent of tinea faciei varies in relation with geographic region and environmental reservoirs.
Although many epidemiologic studies concerning dermatophytosis have been published, only a few large series of tinea faciei have been reported. In Italy, ${ }^{2,8}$ Spain, ${ }^{7}$ Switzerland ${ }^{10}$ and Greece, ${ }^{11}$ the zoophilic species Microsporum canis and the granular forms of Trichophyton mentagrophytes var. granulare were the most frequent isolated agents, in most cases related to close contact with animals.

The aim of this study was to analyse the epidemiologic, clinical and mycological features of tinea faciei cases observed over a 9-year period at the Dermatology and Venerology Department of Hospital Santo António dos Capuchos (Lisbon, Portugal).

\section{MATERIAL AND METHODS}

We undertook a retrospective study of all patients with facial lesions suggestive of tinea faciei who underwent mycological tests (direct examination and culture) from January 2008 to December 2016 at the 

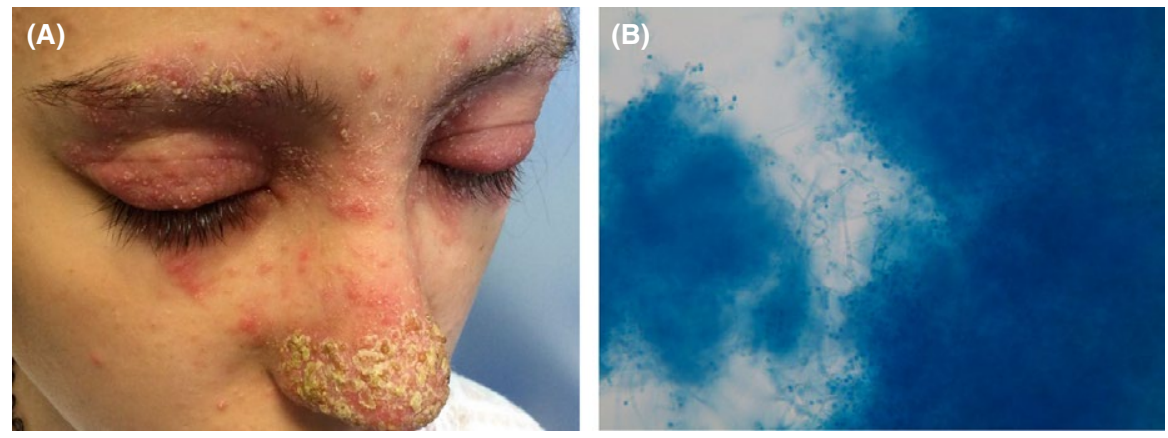

FIGURE 1 A, Tinea faciei on a child with daily contact with a rabbit. B, Microscopic examination with lactophenol blue solution from the colony revealed abundant microconidia in dense clusters and spirals hyphaes, findings typical of Trichophyton mentagrophytes var. granulare

Department of Dermatology and Venerology of Hospital Santo António dos Capuchos, Lisbon, Portugal. Data regarding demographic, clinical and mycological characteristics were obtained from clinical charts.

Direct microscopic examination of the samples, including scale, was performed using potassium hydroxide (20\%). Mycological cultures were incubated at $24^{\circ} \mathrm{C}$ in Mycobiotic agar ${ }^{\circledR}$ and re-evaluated periodically for at least 3 weeks.

Considering that the present study used pre-existing data, recorded anonymously in a database not specifically for any study purpose, the usage of such data was approved, without the need for obtaining informed consent near the patients.

\section{3 | RESULTS}

During a period of 9 years, 72 patients were diagnosed with tinea faciei, corresponding to a $7.5 \%$ of the total (963) of glabrous skin tinea (comprising all anatomic locations). Of these 72 patients, 37 (51.4\%) were male and 35 (48.6\%) female. Forty-three cases (59.7\%) occurred in children younger than 12 years, 7 (9.7\%) between 12 and 18 years, 10 (13.9\%) between 18 and 40 years and 12 (16.7\%) were older than 40 years.

Concerning clinical presentation, inflammatory forms of tinea were observed in 6 (8.3\%) patients, being 3 children (Figure 1) and 3 adults. One quarter of the patients were also affected by tinea infection in other areas of the body, 8 (11.1\%) of which being diagnosed with tinea capitis ( 6 children and 2 adults). Ten patients (13.8\%) presented with tinea incognito, and mentioned previous treatment with topical steroids as a result of misdiagnosis.

Direct microscopic examination was positive in 59 (81.9\%) of 72 specimens. Culture was positive in $70(97.2 \%)$ cases, $53(75.7 \%)$ of which corresponded to anthropophilic, 15 (21.4\%) to zoophilic and 2 (2.9\%) to geophilic dermatophytes.

Microsporum audouinii was isolated in 16 (22.3\%) cases, Trichophyton soudanense in 15 (21.4\%) cases and T. rubrum was the causative agent in 14 (20.0\%) cases. Trichophyton mentagrophytes (always var. granulare), M. canis, T. tonsurans, M. gypseum, M. ferrugineum and T. violaceum were also isolated in few cases (Table 1).

Concerning the investigation of the infection source, only in 6 cases ( 4 adults and 2 children) we could identify contact with infected pets such as cats, dogs and rabbits.

Most of the patients (52\%) were treated with systemic antifungals, and the remaining were treated with topical therapies only. Reasons for systemic treatment included the involvement of vellus hair, multiple lesions and concomitant dermatophytosis in other areas.

All the patients had a complete resolution of skin lesions with no side effects observed.

\section{4 | DISCUSSION}

We report a large series of tinea faciei along a 9-year study period that allow us to make some epidemiological and clinical considerations.

Some authors consider tinea faciei to be a rare dermatophytosis. ${ }^{8}$ However, our series of 72 cases (representing $7.5 \%$ of the total of tinea corporis cases) diagnosed in a relatively short period of time shows that it is not such a rare entity, at least in our population. It remains to be clarified with further studies whether this is due to under- or misdiagnosis (as in previous published studies), or if we have

TABLE 1 Tinea faciei epidemiological and mycological features

\begin{tabular}{|lc|}
\hline \multicolumn{1}{|l}{} & $\mathrm{n}(\%)$ \\
\hline Gender & \\
\hline Male & $37(51.4)$ \\
\hline Female & $35(48.6)$ \\
\hline Age $(\mathrm{y})$ & \\
\hline$<12$ & $43(59.7)$ \\
\hline $13-17$ & $7(9.7)$ \\
\hline $18-39$ & $10(13.9)$ \\
\hline$>40$ & $12(16.7)$ \\
\hline Mycological examination & \\
\hline Direct positive & $59(81.9)$ \\
\hline Culture positive & $70(97.2)$ \\
\hline Culture & \\
\hline Microsporum audouinii & $16(22.3)$ \\
\hline Trichophyton soudanense & $15(21.4)$ \\
\hline Trichophyton rubrum & $14(20.0)$ \\
\hline Trichophyton mentagrophytes var. granulare & $9(12.9)$ \\
\hline Trichophyton tonsurans & $6(8.6)$ \\
\hline Microsporum canis & $6(8.6)$ \\
\hline Microsporum gypseum & $2(2.9)$ \\
\hline Microsporum ferrugineum & $1(1.4)$ \\
\hline Trichophyton violaceum & $1(1.4)$ \\
\hline
\end{tabular}


TAB LE 2 Tinea faciei mycological examination; differences with no statistical significance $(\kappa=-0.032 ; 95 \% \mathrm{Cl})$

\begin{tabular}{llll} 
& \multicolumn{2}{l}{ Culture } \\
\cline { 2 - 4 } Direct microscopy & Positive & Negative & Total \\
\hline Positive & 57 & 2 & 59 \\
Negative & 13 & 0 & 13 \\
\hline Total & 70 & 2 & 72 \\
\hline
\end{tabular}

a higher incidence in our population when compared to other epidemiologic settings.

Of the 72 confirmed tinea faciei cases, 2 (2.8\%) cases revealed fungi structures in direct microscopic examination, but there was no fungal growth in the culture, probably related to previous treatment with topic antifungal in both cases. On the other hand, 13 (18.1\%) had positive culture results but were negative in direct microscopic examination, mostly due to sample scarcity (Table 2 ).

It has been suggested that females are more frequently affected than males. However, considering that facial dermatophyte infections in males are often diagnosed as tinea barbae, whereas in females are diagnosed as tinea faciei, this difference is probably not real. ${ }^{1}$ In fact, in our study no statistically significant difference between sex groups was found.

In contrast to other reports that found 2 age peaks of incidence, ${ }^{8}$ in our series, most cases (59.72\%) occurred in children. The association between tinea faciei and tinea capitis in children has been described previously. ${ }^{9}$ Additionally, the epidemiology of tinea capitis has changed over the past years with an increase in anthropophilic infections primarily linked to patients of African ethnicity. ${ }^{10}$ According to that, we hypothesise that, as previously described for tinea capitis, increased migration is contributing to change the epidemiology of tinea faciei in our geographic area. ${ }^{12}$

Also in contrast to other studies, ${ }^{1,3,7,13}$ which found a prevalence of up to $30 \%$ of tinea incognito in the face, we observed a lower frequency (13.89\%) of tinea incognito in this anatomic location (Figure 2). This is probably associated with the greater restrictions in primary care referrals to specialists, and the lack of understanding of this clinical entity by those clinicians. The facial anatomy, exposure

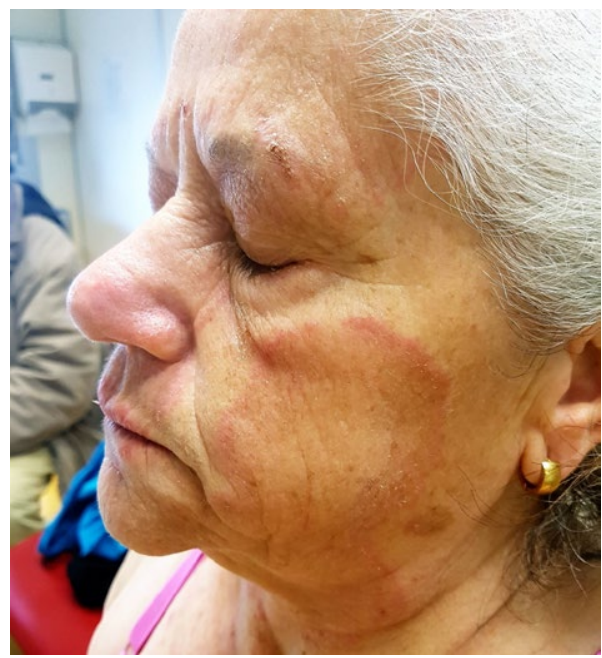

FIGURE 2 Tinea incognito as a result of tinea faciei treated with topical steroids for 2 weeks to sunlight and frequent use of cosmetics often determine atypical clinical presentation, ${ }^{8}$ which highlights the importance of the tinea faciei in differential diagnosis of facial dermatosis.

Systemic treatment was prescribed to most (52\%) of our patients in association with topical antifungals in order to achieve clinical resolution. In fact, topical antifungals are frequently insufficient because of the involvement of vellus hair by the dermatophyte, resulting in need of oral antifungals. ${ }^{2,8}$

The main limitation of this study was its retrospective design, which restrains the clinical, microbiological and epidemiological characterisation. More prospective studies with a larger number of patients are needed to better understand the clinical and epidemiological features of tinea faciei.

\section{CONFLICT OF INTEREST}

None declared.

\section{ORCID}

Ana Borges (iD http://orcid.org/0000-0002-2965-5962

Ana Brasileiro iD http://orcid.org/0000-0002-8359-6561

\section{REFERENCES}

1. Lin RL, Szepietowski JC, Schwartz RA. Tinea faciei, an often deceptive facial eruption. Int J Dermatol. 2004;43:437-440.

2. Romano C, Ghilardi A, Massai L. Eighty-four consecutive cases of tinea faciei in Siena, a retrospective study (1989-2003). Mycoses. 2005; 48:343-346.

3. Jorquera E, Moreno JC, Camacho F. Tinea faciei: epidemiology. Ann Dermatol Venereol. 1992;119:101-104.

4. Meymandi S, Wiseman MC, Crawford RI. Tinea faciei mimicking cutaneous lupus erythematosus: a histopathologic case report. J Am Acad Dermatol. 2003;48(2 Suppl):S7-S8.

5. Gorani A, Schiera A, Oriani A. Case report Rosacea-like Tinea incognito. Mycoses. 2002;45:135-137.

6. Alteras I, Sandbank M, David M, Segal R. 15-year survey of tinea faciei in the adult. Dermatologica. 1988;177:65-69.

7. del Boz J, Crespo V, de Troya M. Pediatric tinea faciei in southern Spain: a 30-year survey. Pediatr Dermatol. 2012;29:249-253.

8. Nicola A, Laura A, Natalia A. A 20-year survey of tinea faciei. Mycoses. 2010;53:504-508.

9. Aly R. Ecology, epidemiology and diagnosis of tinea capitis. Pediatr Infect Dis J. 1999;18:180-185.

10. Kieliger S, Glatz M, Cozzio A, Bosshard PP. Tinea capitis and tinea faciei in the Zurich area - an 8-year survey of trends in the epidemiology and treatment patterns. J Eur Acad Dermatol Venereol 2015;29:1524-1529.

11. Maraki S, Mavromanolaki VE. Epidemiology of Dermatophytoses in Crete, Greece. Med Mycol J. 2016;57:E69-E75.

12. Campos S, Lestre S, Galhardas C, Apetato M. Tinea capitis - a retrospective study of 5 years (2008-2012) in Hospital Santo António dos Capuchos. Revista SPDV. 2014; vol. 72, n.3.

13. Maritati E, Gianni C. Tinea incognito in Italy: a 15-year survey. Mycoses. 2006;49:383-387.

How to cite this article: Borges A, Brasileiro A, Galhardas C, Apetato M. Tinea faciei in a central Portuguese hospital: A 9-year survey. Mycoses. 2018;61:283-285. https://doi.org/10.1111/myc.12730 